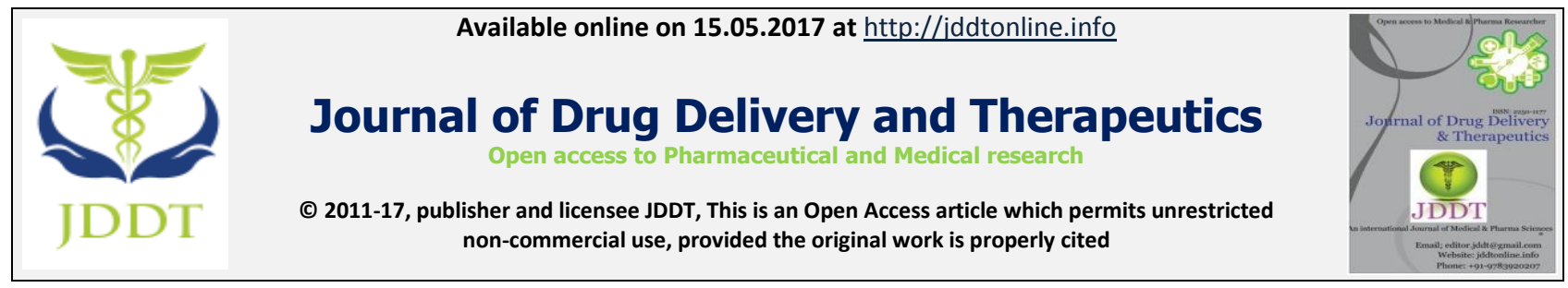

Mini Review

\title{
COMBINATIONAL DRUG DELIVERY OF DOXORUBICIN FOR ENHANCED ANTITUMOR EFFICACY
}

\author{
Hongli Yu $\mathbf{u}^{\mathrm{a}, \mathrm{b}, \mathrm{c}, \mathrm{d}} \mathbf{1}$, Kuilong Wang ${ }^{\mathrm{a}, 1}$, Hao Wu ${ }^{\mathrm{a}, \mathrm{b}, \mathrm{c}, \mathrm{d}^{*}}$, Xingde Zhang ${ }^{\text {a,d,e* }}$

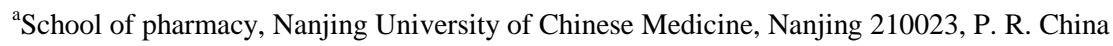 \\ ${ }^{\mathrm{b}}$ Jiangsu Key Laboratory of Chinese Medicine Processing, Nanjing University of Chinese Medicine, Nanjing 210023, P. R. China \\ ${ }^{c}$ Engineering Center of State Ministry of Education for Standardization of Chinese Medicine Processing, Nanjing 210023, P. R. China \\ ${ }^{\mathrm{d} S t a t e}$ Key Laboratory Cultivation Base for TCM Quality and Efficacy, Nanjing University of Chinese Medicine, Nanjing 210023, P. R. \\ China \\ ${ }^{\mathrm{e}}$ Yancheng Traditional Chinese Medicine Hospital Affiliated to Nanjing University of Chinese Medicine \\ ${ }^{1}$ these authors contributed equally to this work
}

Article Info: Received 16 March 2017; Review Completed 24 April 2017; Accepted 05 May 2017, Available online 15 May 2017 Cite this article as:

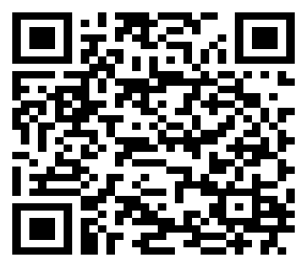

Yu H, Wang K, Wu H, Zhang $\mathrm{X}$, Combinational drug delivery of doxorubicin for enhanced antitumor efficacy, Journal of Drug Delivery and Therapeutics. 2017; 7(3):66-69

DOI: http://dx.doi.org/10.22270/jddt.v7i3.1423

*Address for Correspondence:

Xingde Zhang, Nanjing University of Chinese Medicine, Nanjing-210023, China. Tel.: +86 02586798281. E-mail address: whao5795@163.com

\begin{abstract}
The present study depicts the enhancement of antitumor efficacy and the safety usage of the DOX with the combination of the coenzymes. The drugs were prepared by the diffusion evaporation method of the solvent and were also easily optimized. The therapeutic antitumor efficacy is found to be signification with the combinations undertaken for the study. The improvements of the processes were indicated in the form of the literature review, which was analyzed for the purpose of proving the therapeutic efficacy in the drugs. The nanocarriers' multifunctional characteristics and the importance of the liposome in the treatment of the tumor were focused in this study, which enables the enhancement of the antitumor efficacy.
\end{abstract}

Keywords: Nanomedicine, drug delivery, Doxorubicin, anti-cancer.

\section{INTRODUCTION}

In the field of cancer treatment, large changes can be found. There are several doctors and medical scientists conducting research to know the effective treatment of cancer. ${ }^{1,2}$ Moreover, in this matter, development of doxorubicin drug is significant. Doxorubicin is an important drug, which is used in chemotherapy to treat various types of cancer. ${ }^{3}$ Some of the common forms of cancer, where doxorubicin can be employed efficiently, are bladder cancer, breast cancer, Kaposi's sarcoma, lymphoma as well as acute lymphocytic leukemia. ${ }^{4,5}$ This important drug is mostly sold under the trade names Adriamycin. In the cancer treatment, usages of nano- medicines also play important roles. ${ }^{6}$ The nano-medicine is considered as the medical application of the nanotechnology. ${ }^{7,8}$ The usage of nanomaterials along with organic devices is an effective treatment for tumor and is widely used in cancer treatment as by this process efficiently kills the tumor cells. ${ }^{9}$ Besides this, it is broadly observed that for the Chemotherapy two or multiple drugs are used simultaneously. ${ }^{10,11}$ The cause of multidrug dose is the limitation of a single drug in the treatment of cancer. ${ }^{12-14}$ The usage of a single chemotherapeutic drug is not as much effective as a combination of multi-drug in the anti-tumor treatment. ${ }^{15}$ In addition, the only drugs have some limitations like the development of the resistance of the drug, limited 
administration of clinical uses and high toxicity, multidrug use to be administrated. ${ }^{16}$

\section{Doxorubicin with Pyrrolidine Dithiocarbamate}

For the treatment of cancer, doxorubicin is used with a combination of several other drugs. Moreover, in this case, its conjunction with Pyrrolidine dithiocarbamate (PDTC) is very useful. ${ }^{17}$ Co-administration of Pyrrolidine dithiocarbamate (PDTC) and Doxorubicin via micellar nanoparticles used to be performed to attain actively targeted delivery of the drugs. Apart from this, this combination supports in overcoming multi-drug resistance. ${ }^{18}$ The self-assembled micellar nanoparticles from copolymer folate-chitosan were deployed since the carrier to co-administration of pyrrolidine dithiocarbamate (PDTC) and doxorubicin to attain the aimed doxorubicin release along with $\mathrm{pH}$ reactive drug discharge. Besides this, the process of administrating these drugs combination support in overcoming doxorubicin multidrug resistance. The efficient amalgamation of FA-CS used to be decided by the NMR. The standard size of particles was not adequate to attain longevity throughout the systematic circulation. The lesser CACs in neutral as well as alkalescent situations than an acid $\mathrm{pH}$ probably led to keep the good constancy of micellar nanoparticles in the blood stream. The doxorubicin along with PDTC encapsulating the efficacy of the micellar nanoparticles were 77.64 and $86.54 \mathrm{wt} \%$, whereas the loading substance was 12.34 and $15.32 \%$ correspondingly. The drug doxorubicin at neutral or alkalescent $\mathrm{pH}$ release was slow and constant, but in weak acidic condition, the release was much quicker near to $75-95 \%$ of its whole drug substance.

\section{Doxorubicin with Paclitaxel}

The limitations regarding the antitumor are indicated by the single use of the chemotherapeutic drugs. ${ }^{19,20}$ For the purpose of removing the toxicity amount of the drugs, the mixture of the Paclitaxel and the Doxorubicin is being used for decreasing the toxicity of the drugs used for the treatment of the tumors. Therefore, the mixture of two or more therapeutic drugs was proposed with indicating the decrement of each drug. The delivery of the chemotherapeutic drug is being provided by the aid of the drug carriers which are the micelles, inorganic nanoparticles, and the liposomes. The core shell of the nanoparticles is doubly emulsified from an amphiphilic copolymer Methoxy poly (ethylene glycol)-poly (lactide-co-glycolide) or the mPEG-PLGA. ${ }^{21}$ This aids in creating an advantage for initiation of the fabrication process that can be improved by the process of the double emulsion. The nanoparticles are seemed to be not able to deliver the hydrophilic doxorubicin and the hydrophilic paclitaxel. Therefore, the loading of the drugs is undertaken for the purpose of possessing a better polydispersity, and it also indicates controlled nature of the size distribution. The co-delivery of the nanocarriers are suppressed the tumor cells in a more efficient way than the DOX and the TAX with keeping the same concentrations.

\section{Doxorubicin with siRNA}

The glioma is considered as a fatal malignancy in the brain which cannot be successfully treated with aid of chemotherapy. $^{22,23}$ Thus, the development of various techniques is undertaken for the purpose of providing appropriate treatment for the glioma with considering the clinical importance. On the basis of this factor, the folate functioned multifunctional nanocarrier is created in the lab which is effective for the co-delivering the DOX and the siRNA into the rat C6 glioma cells. ${ }^{24}$ Therefore, the achievement of this experiment created a great success with expressing the folate receptor cells. With the aid of this, the anti-apoptotic responses in the C6 cells were activated and were also revealed by the molecular biological investigations. ${ }^{25}$ The study undertaken in the case of the animals provided an appropriate example for the purpose of indicating a distinct down-regulation of the anti-apoptotic BCL-2 gene. It also showed the outstanding up-regulation of Bax gene which resulted in activation of the caspase- 3 . The up-regulation is also remarkably seen in the cell apoptosis in the tumor tissues. Henceforth, the healing effect can be appropriately achieved with using folatetargeted co-delivery plan.

\section{Doxorubicin along with BCL-2 SIRNA}

Co-delivery or combined administration of DOX along with siRNAs by mesoporous silica nanoparticles were provided into the multidrug-resistance cancer cells for the purpose of achieving the minimum premature discharge. It substantially improves the efficiency of chemotherapy with overcoming the nonpump resistance as well as avoiding the efflux pump resistance. ${ }^{26}$ In this matter, it must be remembered that growth of resistance regarding the multidrug in cancer cells generates the adverse effects that are shown to be the important obstacles for successful chemotherapy. The therapists are trying to develop effective strategies to overcome the drug resistance and to target the particular tumor with a minimum discharge of the premature drug. This particular strategy has an immense impact in the curing process of cancer. The multidrug resistance is a resistance phenotype where the cancer cells turn into resistance simultaneously to multiply the drugs. The apparent structural similarity is not identified as well as the diverse molecular targets are seen. The multidrug resistance or MDR can be segregated into two different classes: pump resistance and non-pump resistance. The causes of pump resistance are few proteins, which make membrane bound adenosine triphosphate (ATP). The key mechanism of non-pump resistance is indicated in the form of activating cellular anti-apoptotic defense initially by $\mathrm{Bcl}-2$ protein. ${ }^{27}$ 


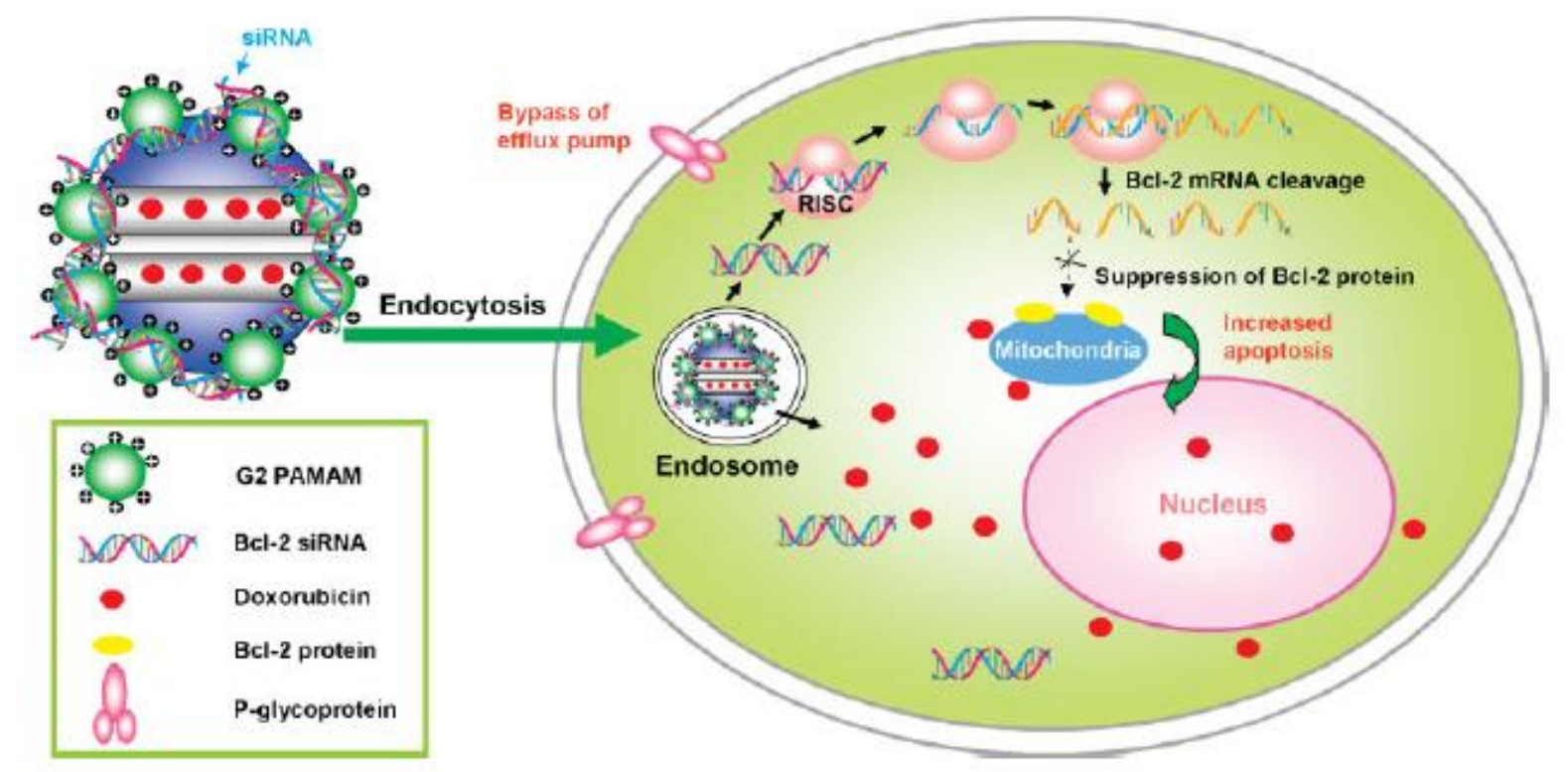

Schematic diagram of co-administration system depends on MSNs for delivering doxorubicin and Bcl-2- target siRNA

\section{Doxorubicin with Verapamil}

For efficient administration of cancer chemotherapy, the greatest obstacle is multidrug resistance (MDR). ${ }^{28-30}$ The multidrug resistance (MDR) is frequently linked with up-regulation of the permeability-glycoprotein (Pgp), which is an ATP-dependent efflux pump and support in decreasing intracellular drug concentration. For overcoming the multidrug resistance (MDR), a range of approaches has been explored, including the usage of cytotoxic drugs combined with Pgp inhibitor. Verapamil (VER) is a calcium channel adversary has been exhibit Pgp inhibitory activity. However, the amount of dose of VER needed for Pgp blockade (2$6 \mu \mathrm{M})$ is substantially higher in comparison to the present clinical treatment for arrhythmia and possibly induce cardiotoxicity. In this matter, it is observed that liposomal delivery of VER can decrease its cardiotoxicity by declining free VER concentration in the systemic circulation. ${ }^{31}$ It is observed that liposomes along with doxorubicin and verapamil (VER) and mixed with transferring (Tf-L-DOX/VER) were produced as well as examined in K562 Leukemia Cells. The formulation is to target the tumor cells, decreasing cardiotoxicity of Doxorubicin and Verapamil. Besides this, this combination support in overcoming Pglycoprotein (Pgp) mediated multidrug resistance phenotype. The mixer of TfR targeting, coencapsulation of doxorubicin and verapamil is shown to be highly efficient for overcoming the drug resistance in the K562 leukemia cell.

\section{SUMMARY}

The combined administration of drugs in cancer therapy is most effective. Moreover, for the treatment of a tumor, the drug combinations are used effectively. ${ }^{32}$ Mostly doxorubicin drug is utilized in the treatment of cancer or tumor along with other drugs. Along with doxorubicin, several drugs like Pyrrolidine dithiocarbamate (PDTC), Paclitaxel, Sirna by multifunctional nanocarrier, Bcl-2 Sirna by Mesoporous Silica Nanoparticles, and Verapamil via transferrin liposome very useful in the treatment of cancer, specifically for tumor treatment. It supports in reducing the multidrug resistance. 


\section{REFERENCES}

1. Etheridge M L, Campbell S A, Erdman A G, Haynes C L, Wolf $\mathrm{S} \mathrm{M}$, and McCullough $\mathrm{J}$. The big picture on nanomedicine: the state of investigational and approved nanomedicine products. Nanomedicine: nanotechnology, biology and medicine. 2013, 9: $1-14$

2. Cheng X. Developing organic and inorganic nanomedicine for cancer therapy. Journal of Drug Delivery and Therapeutics. 2017, 7(3): 1-4 DOI: 10.22270/jddt.v7i2.1367

3. Cheng X, and Lee R J. The role of helper lipids in lipid nanoparticles (LNPs) designed for oligonucleotide delivery. $A d v$ Drug Deliv Rev. 2016, 99: 129-137; doi: 10.1016/j.addr.2016.01.022.

4. Jain K. Future of nanomedicine: impact on healthcare \& society. 2015 ,

5. Cheng X, Liu Q, Li H, Kang C, Liu Y, Guo T, et al. Lipid Nanoparticles Loaded with an Antisense Oligonucleotide Gapmer Against Bcl-2 for Treatment of Lung Cancer. Pharm Res. 2017, 34: 310-320; doi: 10.1007/s11095-016-2063-5.

6. Sun Y, Kang C, Liu F, and Song L. Delivery of Antipsychotics with Nanoparticles. Drug Development Research. 2016, 77: 393-399

7. Kang C, Sun Y, Wang M, and Cheng X. Nanosized camptothecin conjugates for single and combined drug delivery. European Journal of BioMedical Research. 2016, 2: 8-14

8. Kang C, Sun Y, Zhu J, Li W, Zhang A, Kuang T, et al. Delivery of Nanoparticles for Treatment of Brain Tumor. Current Drug Metabolism. 2016, 17: 745-754

9. Liu F, Sun Y, Kang C, and Zhu H. Pegylated Drug Delivery Systems: From Design to Biomedical Applications. Nano LIFE. 2016, 6: 1642002

10. Sumer B, and Gao J. Theranostic nanomedicine for cancer. Nanomedicine. 2008, 3: 137-140

11. Kang C, Qin J, Osei W, and Hu K. Regulation of protein kinase $\mathrm{C}$-epsilon and its age-dependence. Biochemical and Biophysical Research Communications. 2017, 482: 1201-1206

12. Sun Y, Kang C, Yao Z, Liu F, and Zhou Y. Peptide-Based Ligand for Active Delivery of Liposomal Doxorubicin. Nano LIFE. 2016, 6: 1642004

13. Sun Y, Kang C, Zhang A, Liu F, Hu J, Zhong X, et al. Codelivery of dual-drugs with nanoparticle to overcome multidrug resistance. European Journal of BioMedical Research. 2016, 2 12-18

14. Waller A P, George M, Kalyanasundaram A, Kang C, Periasamy M, Hu K, et al. GLUT12 functions as a basal and insulin-independent glucose transporter in the heart. Biochimica et Biophysica Acta (BBA)-Molecular Basis of Disease. 2013, 1832: $121-127$

15. Chen Y, Bian Y, Sun Y, Kang C, Yu S, Fu T, et al. Identification of 4-aminoquinoline core for the design of new cholinesterase inhibitors. PeerJ. 2016, 4: e2140

16. Han R, Sun Y, Kang C, Sun H, and Wei W. Amphiphilic dendritic nanomicelle-mediated co-delivery of 5-fluorouracil and doxorubicin for enhanced therapeutic efficacy. Journal of Drug Targeting. 2017, 25: 140-148

17. Fan L, Li F, Zhang H, Wang Y, Cheng C, Li X, et al. Codelivery of PDTC and doxorubicin by multifunctional micellar nanoparticles to achieve active targeted drug delivery and overcome multidrug resistance. Biomaterials. 2010, 31: 56345642; doi: 10.1016/j.biomaterials.2010.03.066.

18. Qiao H, Fang D, Chen J, Sun Y, Kang C, Di L, et al. Orally delivered polycurcumin responsive to bacterial reduction for targeted therapy of inflammatory bowel disease. Drug Delivery. 2017, 24: 233-242
19. Song L, Kang C, Sun Y, Huang W, Liu W, and Qian Z. Crocetin Inhibits Lipopolysaccharide-Induced Inflammatory Response in Human Umbilical Vein Endothelial Cells. Cellular Physiology and Biochemistry. 2016, 40: 443-452

20. Sun Y, and Kang C. Self-Assembly of Peptides into Hydrogel. Journal of Organic \& Inorganic Chemistry. 2016,

21. Wang $\mathrm{H}$, Zhao Y, Wu Y, Hu Y L, Nan K, Nie G, et al. Enhanced anti-tumor efficacy by co-delivery of doxorubicin and paclitaxel with amphiphilic methoxy PEG-PLGA copolymer nanoparticles. Biomaterials. 2011, 32: 8281-8290; doi: 10.1016/j.biomaterials.2011.07.032.

22. Duan Y, Pei K, Cai H, Tu S, Cheng X, Zhang Z, et al. Strategy of integrated evaluation on treatment of traditional Chinese medicine as 'interaction of system to system'and establishment of novel fuzzy target contribution recognition with herb-pairs, a case study on Astragali Radix-Fructus Corni. Molecular and Cellular Endocrinology. 2016, 434: 219-237

23. Duan Y, Pei K, Cai H, Tu S, Zhang Z, Cheng X, et al. Bioactivity evaluation-based ultra high-performance liquid chromatography coupled with electrospray ionization tandem quadrupole-time-of-flight mass spectrometry and novel distinction of multi-subchemome compatibility recognition strategy with Astragali Radix-Fructus Corni herb-pair as a case study. Journal of pharmaceutical and biomedical analysis. 2016, 129: 514-534

24. Cheng D, Cao N, Chen J, Yu X, and Shuai X. Multifunctional nanocarrier mediated co-delivery of doxorubicin and siRNA for synergistic enhancement of glioma apoptosis in rat. Biomaterials. 2012, 33: 1170-1179; doi: 10.1016/j.biomaterials.2011.10.057

25. Li H, Cheng X, Liu Y, Lee Y B, Kim D J, Ahn C-h, et al. Folate receptor-targeted lipid coated albumin nanoparticles (F-LCAN) for therapeutic delivery of RX-0201 (Archexin $®$ ), an antisense oligonucleotide against Akt-1. 2016,

26. Li H, Quan J, Zhang M, Yung B C, Cheng X, Liu Y, et al. Lipid-Albumin Nanoparticles (LAN) for Therapeutic Delivery of Antisense Oligonucleotide against HIF-1alpha. Mol Pharm. 2016, 13: 2555-2562; doi:

10.1021/acs.molpharmaceut.6b00363

27. Chen A M, Zhang M, Wei D, Stueber D, Taratula O, Minko T, et al. Co-delivery of doxorubicin and $\mathrm{Bcl}-2$ siRNA by mesoporous silica nanoparticles enhances the efficacy of chemotherapy in multidrug-resistant cancer cells. Small. 2009, 5: 2673-2677; doi: 10.1002/smll.200900621.

28. XU S-h, Chen K, CHEN M-l, ZHOU P-p, HE G-w, CUI Y-j, et al. Dynamic expression of AQP4 in early stageof ischemia/reperfusion rats and cerebral edema. Chinese Pharmacological Bulletin. 2016: 1433-1441

29. Xue X, Zhao N-Y, Yu H-T, Sun Y, Kang C, Huang Q-B, et al. Discovery of novel inhibitors disrupting HIF-1 $\alpha /$ von HippelLindau interaction through shape-based screening and cascade docking. PeerJ. 2016, 4: e2757

30. Yang Z, Xie J, Zhu J, Kang C, Chiang C, Wang X, et al. Functional exosome-mimic for delivery of siRNA to cancer: in vitro and in vivo evaluation. Journal of Controlled Release. 2016, 243: 160-171

31. Wu J, Lu Y, Lee A, Pan X, Yang X, Zhao X, et al. Reversal of multidrug resistance by transferrin-conjugated liposomes coencapsulating doxorubicin and verapamil. J Pharm Pharm Sci. 2007, 10: 350-357

32. Yao Z, Sun Y, and Kang C. Structure and Self-Assembly of Multicolored Naphthalene Diimides Semiconductor. Nano LIFE. 2016, 6: 1642007 